\title{
Atypical Aicardi-Goutieres Syndrome: Is the WRN Locus a Modifier?
}

\section{Davor Lessel, ${ }^{2}$ Bidisha Saha, ${ }^{1}$ Fuki Hisama, ${ }^{3}$ Bahar Kaymakamzade, ${ }^{4}$ Gulay Nurlu, ${ }^{5}$ Yasemin Gursoy-ÖZzemir, ${ }^{5,6}$ Holger Thiele, ${ }^{7}$ Peter Nürnberg, ${ }^{7,8,9}$ George M. Martin, ${ }^{1}$ Christian Kubisch, ${ }^{2}$ and Junko Oshima ${ }^{1 *}$}

${ }^{1}$ Department of Pathology, University of Washington, Seattle, Washington

${ }^{2}$ Institute of Human Genetics, University of UIm, Ulm, Germany

${ }^{3}$ Division of Medical Genetics, Department of Medicine, University of Washington, Seattle, Washington

${ }^{4}$ Department of Neurology, Faculty of Medicine, Near East University, Cyprus

${ }^{5}$ Department of Neurology, Hacettepe University, Ankara, Turkey

${ }^{6}$ Institute of Neurological Sciences and Psychiatry, Hacettepe University, Ankara, Turkey

'Cologne Center for Genomics, University of Cologne, Cologne, Germany

${ }^{8}$ Center for Molecular Medicine Cologne, University of Cologne, Cologne, Germany

${ }^{9}$ Cologne Excellence Cluster on Cellular Stress Responses in Aging-Associated Diseases, University of Cologne, Cologne, Germany

Manuscript Received: 1 March 2014; Manuscript Accepted: 2 May 2014

We describe a 28-year-old Turkish man with consanguineous parents who presented with an aged appearance with prematurely gray hair and scleroderma-like skin, spastic paraplegia, and apparent disability. The proband and each of his parents were heterozygous for a mutation in $W R N$, which could not explain his symptoms. Exome sequencing of the proband's blood DNA showed a homozygous c.626-1G $>$ C mutation in intron 5 of the SAMHD1 gene, which encodes a triphosphohydrolase involved in the regulation of intracellular $\mathrm{dNTP}$ pools and which is mutated in Aicardi-Goutieres syndrome. The RNA studies confirmed aberrant splicing of exon 6, and family studies showed that both parents are heterozygous for this mutation. We conclude that mutations in SAMHD1 - in addition to causing an early-onset form of encephalopathy in Aicardi-Goutieres syndrome - may present with modest signs of accelerated aging similar to Werner syndrome. The extent to which heterozygosity at the WRN locus may modify the effect of biallelic SAMHD1 mutations is unknown. It is conceivable that synergistic effects of these two mutations might be responsible for the unusual phenotype. (c) 2014 Wiley Periodicals, Inc.

Key words: Werner syndrome; Aicardi-Goutieres syndrome; SAMHD1; WRN; molecular genetics; Mendelian disease; neurology

\section{INTRODUCTION}

Aicardi-Goutieres syndrome (AGS) is a progressive neurological disorder characterized by early onset encephalopathy, intellectual disability, and intracranial calcification involving the basal ganglia [Stephenson, 2008]. The International Registry of Werner Syndrome
How to Cite this Article:

Lessel D, Saha B, Hisama F,

Kaymakamzade B, Nurlu G, Gursoy-

Özdemir Y, Thiele H, Nürnberg P, Martin

GM, Kubisch C, Oshima J. 2014. Atypical

Aicardi-Goutieres syndrome: Is the WRN

locus a modifier?.

Am J Med Genet Part A. 164A:2510-2513.

has been recruiting patients with progeroid features since 1988 from all over the world, primarily based on the clinical diagnostic criteria of Werner syndrome (WS). Classical WS is caused by biallelic mutations in the WRN gene that encode a nuclear helicase and plays a role in maintenance of genomic stability [Friedrich et al., 2010; Oshima et al., 2014]. Here, we report a family from Turkey referred to our Registry because of overlapping features of WS in which we identified both a novel homozygous null mutation of $S A M D H 1$, a locus that encodes a triphosphohydrolase and regulates intracellular dNTP pools, as well as a heterozygous WRN mutation. This research

Grant sponsor: NIH; Grant number: R24AG042328; Grant sponsor: Ellison Medical Foundation; Grant number: AG-SS-1915-07.

${ }^{*}$ Correspondence to:

Junko Oshima, MD, PhD, FACMG, Department of Pathology, University of Washington, Box357470, HSB K-543, Seattle, WA 98195-7470, USA

E-mail: picard@u.washington.edu

Article first published online in Wiley Online Library

(wileyonlinelibrary.com): 2 July 2014

DOI 10.1002/ajmg.a.36664 
project was reviewed and approved by the Institutional Review Board (45 CFR 46. 102(f)).

\section{CLINICAL REPORT}

The patient was a 29-year-old Turkish man (Fig. 1A) whose birth length $(53 \mathrm{~cm})$ and birth weight $(3.4 \mathrm{~kg})$ were normal. Gait difficulty began at age 2 years, and school performance was affected by age 10-12 years. At age 28, he was found to have apparent intellectual disability, spastic paraparesis, hyperreflexia, and basal ganglia calcifications with cerebral atrophy on cranial CT scans (Fig. 1B). His height was $150 \mathrm{~cm}$ (Z score -3.7$)$, weight was $62 \mathrm{~kg}$ (Z score -0.85), OFC was normal at $56 \mathrm{~cm}$, and blood pressure was $120 / 80$. He had prematurely graying hair, a hoarse voice, small testes and hyperkeratoses of the soles of the feet. Cataracts were absent by slit lamp exam. His fasting blood glucose and lipids were normal. Platelet count was normal $\left(212,000 / \mathrm{m}^{3}\right)$ and liver function tests were mildly elevated (less than two fold). Cerebrospinal fluid (CSF) protein was $34 \mathrm{mg} / \mathrm{dL}$; CSF RBCs and WBCs were not detected. Chilblains of the extremities were absent. Osteoporosis was present on vertebral X-rays. Electromyogram and nerve conduction velocity tests showed evidence of axonal neuropathy and myopathy. A muscle biopsy was normal (Supp. Table I in supporting information online).

His parents were first cousins and of normal stature (mother's height $155 \mathrm{~cm}$, father's height $170 \mathrm{~cm}$ ) (Fig. 2A). An older brother had short stature (adult height $138 \mathrm{~cm}, \mathrm{Z}$ score -5.3 ), diabetes, and general features of accelerated aging (prematurely gray hair and skin wrinkles) and was thought to have WS. He died at 21 years from a cerebral hemorrhage. DNA samples were not available from this brother.

\section{RESULTS}

Sequencing of the coding region of WRN (GenBank \# NG_008870.1, NM_000553.4 and NP_000544.2) in the proband showed a heterozygous c.3493C $>$ T substitution in exon 30; which predicts p.Q1165X (Fig. 2A). There was no evidence for a second mutation at the WRN locus. This alteration had been previously reported as a potential founder mutation; it was observed exclusively among Turkish patients with WS [Friedrich et al., 2010]. Both parents were also heterozygous for the c.3493C $>\mathrm{T}$ mutation. Western blot analysis of the WRN protein from the lymphoblastoid cell lines (LCL) derived from the patient and the parents showed the presence of WRN protein whose size was in agreement with the sequencing results (data not shown).

Because of the consanguinity of the parents, we suspected the presence of a biallelic mutation at another locus and conducted exome sequencing of the proband (Supplementary Methods in supporting information online). We focused our analysis on rare homozygous missense, nonsense, frameshift and splice-site alterations. Using a filter of MAF $<0.01$, bioinformatic filtering of variants identified 307 uncommon SNPs, 21 homozygous variants and 3 protein truncating or splice variants. Two of the latter, c. $259 \mathrm{C}>\mathrm{T}$, p.Arg87Ter (rs118095359) in CST9 and c.861+5G $>\mathrm{A}$ (rs192663616) in GLDC were annotated in the Single Nucleotide Polymorphism Database (dbSNP), leaving one non-annotated sequence change with a severe impact on protein structure: a homozygous c.626-1G>C alteration of SAMHD1 (GenBank\# NG_017059.1, NM_015474.3 and NP_056289.2) (Fig. 2B and C and Supplementaty Table II), a locus known to be mutated in Aicardi-Goutieres syndrome-5 (AGS5; OMIM\# 612952) [Rice et al., 2009]. Sanger sequencing confirmed the homozygous alteration in the proband and showed that both parents were heterozygous for this alteration.

The RT-PCR analyses of SAMHD1 mRNA spanning exons 4 through 8 showed products slightly larger than the wild type product in the patient sample (Fig. 2D). Sequencing of the purified RT-PCR product showed an aberrantly spliced product containing an 18bp insertion including a novel stop codon

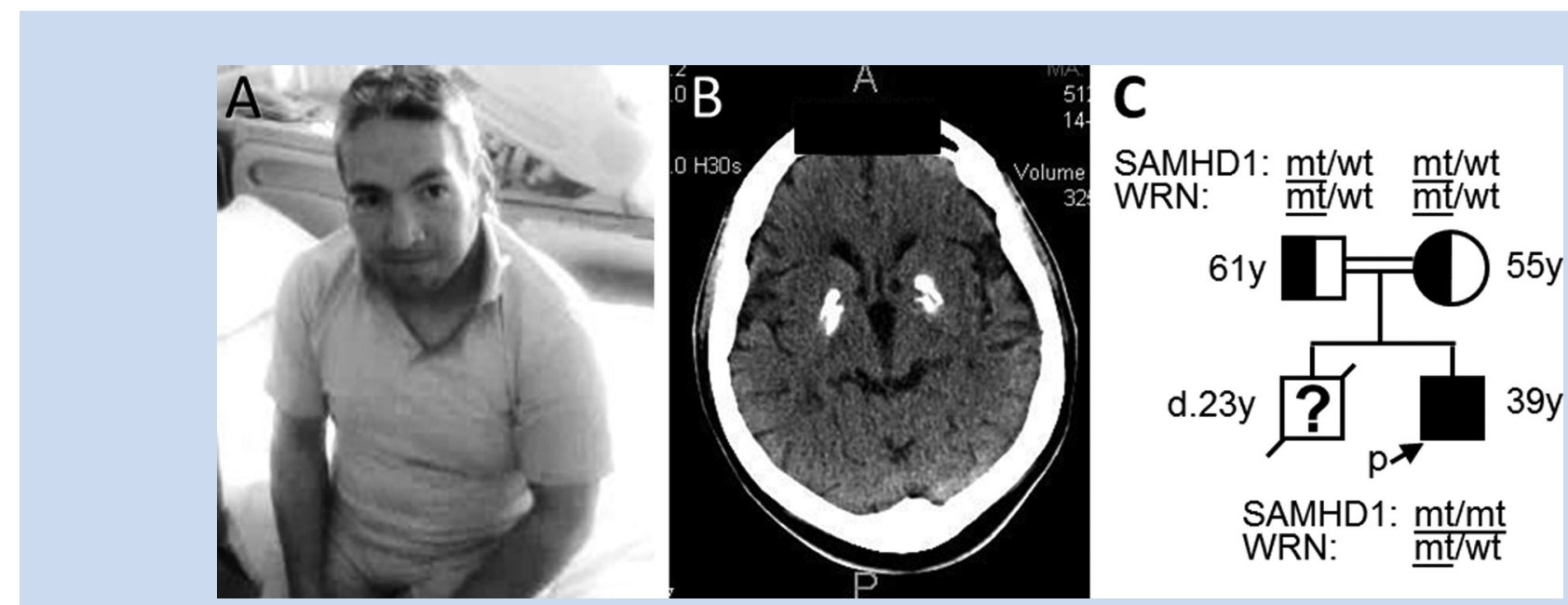

FIG. 1. Patient profile. A) Picture of the patient at age 28 showing prematurely gray hair and overall aged appearance. B] Cranial CT scan of the patient showing calcifications of the basal ganglia and cerebral atrophy. C) The patient's pedigree is shown with SAMHD1 and WRN genotypes. 
A

WRN Exon 30

AlaArgGlnLys $\overline{\mathrm{GCT}} \overline{\mathrm{AGG}} \overline{\mathrm{CAG}} \overline{\mathrm{AAA}}$

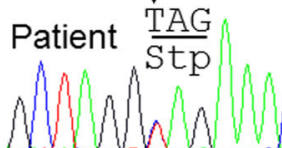

Father

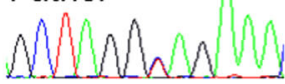

Mother

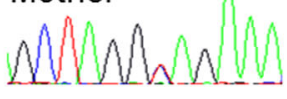

B

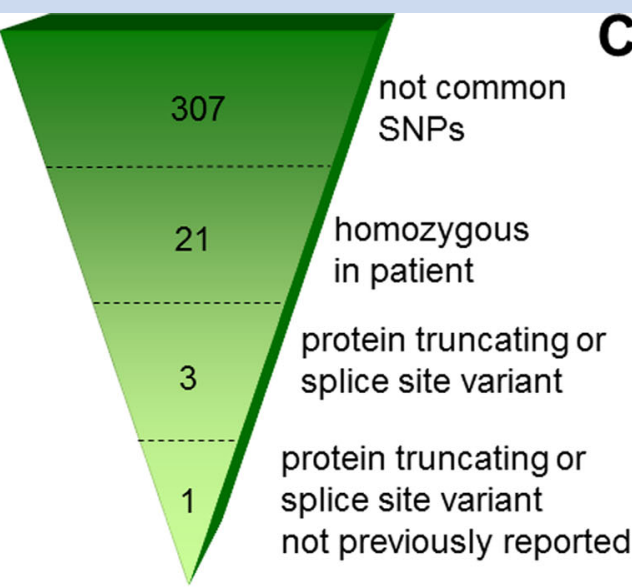

C

SAMHD1

TTTTAACGTCAT Intron $5 \downarrow$ Exon 6
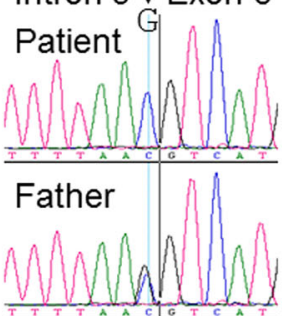

Mother
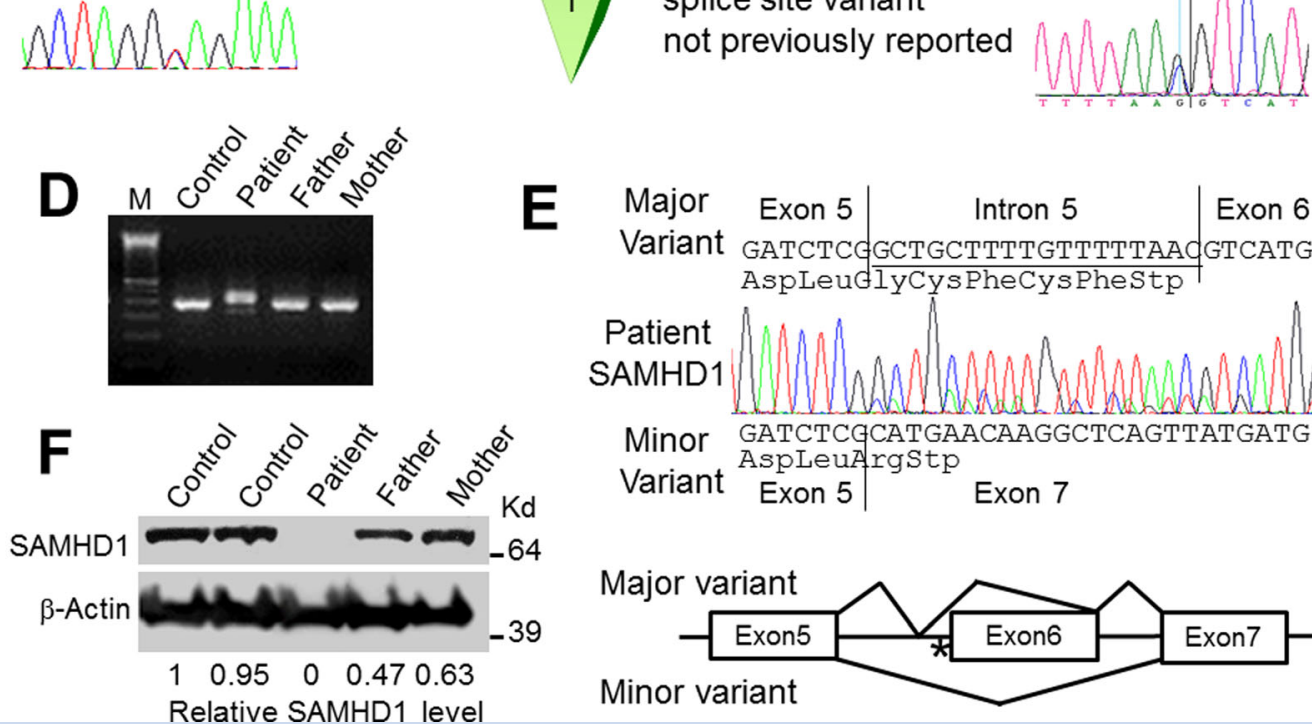

E
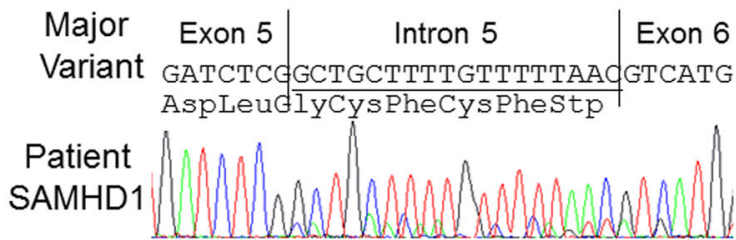

Minor GATCTCGCATGAACAAGGCTCAGTTATGATG

Variant $\begin{gathered}\text { AspLeuA rgStp } \\ \text { Exon } 5\end{gathered}$

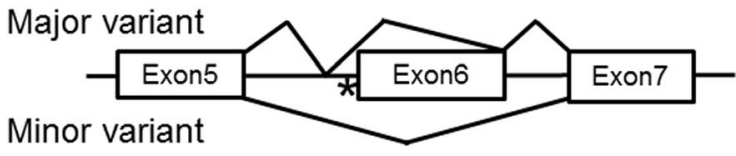

FIG. 2. Molecular studies. A] Evidence of a heterozygous WRN mutation in the patient and his parents. The heterozygous mutation, c.3493C $>T$ [ $p .01165 X$ ], is shown by the arrow. B] A summarization of the exome sequencing performed with patient's DNA. C] Confirmations of SAMHD1 mutations, c.626-1G > C, in the patient and his parents. D) RT-PCR study of SAMHD1 exons 4-8. M indicates the123bp ladder marker. E] The RT-PCR sequencing of exons 4-8 from the patient's RNA. Bottom diagram shows the location of the homozygous mutation ( ${ }^{*}$ ] and the structures of two splice variants. F] Western analysis of SAMHD1 using an anti-N terminal antibody. Relative expression levels of wildtype SAMHD1 were calculated after normalization to $\beta$-actin.

(r.626_627ins18; p.H210Sf*5). Sequencing of the unpurified RTPCR product showed a minor RT-PCR product with a deletion of exon 6 (r.627_697del71; p.G209Af*2) (Fig. 2E). Based on the peak heights of sequences and RT-PCR intensities, the ratios of major and minor splice variants in the patient sample were estimated to be $5 \sim 10$ to 1 . The RT-PCR product of the mutant alleles in LCLs seemed fainter than those of wild type alleles in control LCLs (Fig. 2D), possibly because of nonsense mediated mRNA decay of mutant RNA. Western analysis of SAMHD1 confirmed the absence of wild type protein in the patient and reduced levels in the parents (47 and $63 \%$ of controls) (Fig. 2F).

\section{DISCUSSION}

This is an unusual family in which the proband was found to be a heterozygous carrier of a WRN disease mutation in addition to having inherited null mutations at the SAMHD1 locus from each parent. While cells derived from WRN mutant heterozygotes have been shown to have intermediate sensitivities to genotoxic agents, [Ogburn et al., 1997], individuals with heterozygous WRN mutations do not present with any of the signs and symptoms associated with the Werner syndrome [Oshima et al., 2014]. Immunostaining of 53BP1 in LCLs showed no significant differences in the number of double strand break foci among LCLs from the patient, parents and controls under standard culture conditions (data not shown). These preliminary results raise the possibility that, unlike WS, genomic instability may not be the dominant pathogenetic mechanism in this patient.

Aicardi-Goutieres syndrome is genetically heterogeneous [Stephenson, 2008]. Causative mutations in the TREX1 gene encoding the $3^{\prime} \rightarrow 5^{\prime}$ TREX1 exonuclease or in the three components of the RNASEH2 endonuclease complex (RNASEH2B, RNASEH2C, RNASEH2D) account for $85 \%$ of cases (AGS1-AGS4 respectively). Mutations in SAMHD1 are found in approximately 
$5 \%$ of patients with AGS. There are two typical clinical presentations associated with mutations in these genes: a neonatal onset resembling congenital infection, and a later onset beginning after a period of normal neurological development. The typical neurological phenotype includes microcephaly, seizures, dystonic posturing, and severe intellectual disability. Hepatomegaly, thrombocytopenia and chilblains are frequent. A chronic CSF lymphocytosis and intracranial calcifications are characteristic. Death by age 10 years is common, although there is significant variability [Rice et al., 2009].

One of the puzzling features of AGS is a pronounced phenotypic variability - quite unusual for autosomal recessive conditions, particularly in patients with $S A M H D 1$ or RNASEH2B mutations. Phenotypes associated with $S A M H D 1$ mutations range from "classical" presentation [Rice et al., 2009] to mild intellectual disability with a non-specific chronic inflammatory skin condition [Dale et al., 2010]. There are a series of reports describing cerebral vascular complications that are primarily occlusive and inflammatory in nature which result in variable degrees of neurological dysfunction [Thiele et al., 2010; Xin et al., 2011]. Cerebral vasculopathy with a very wide range of severity was observed within a highly genetically homogeneous Amish community [Xin et al., 2011]. Although we do not have sufficient clinical information or biological samples from the deceased brother of our patient, it is possible that his cerebral hemorrhage was due to the same SAMND1 mutation and that this family is an example of variable expressivity.

SAMHD1 (SAM domain- and HD domain-containing protein 1) encodes a deoxynucleotide triphosphohydrolase which inhibits reverse transcription by reducing intracellular pools of dNTPs [Lahouassa et al., 2012]. SAMHD1 also possesses $3^{\prime} \rightarrow 5^{\prime}$ exonuclease activity, which can cleave viral and endogenous nucleic acids [Beloglazova et al., 2013]. SAMHD1 plays a role in the restriction of HIV-1 (human immunodeficiency virus -1) and in the suppression of autoimmune responses [Beloglazova et al., 2013]. Loss of SAMHD1 activity results in the cellular accumulation of nucleic acids, which is thought to enhance autoimmune responses.

It has been reported that $S A M H D 1$ is commonly mutated in chronic lymphocytic leukemia, likely because of its role in the regulation of cell proliferation and survival, and in the response to DNA damage [Clifford et al., 2013]. These studies suggest that the accumulation of DNA damage might play a significant role in the development of AGS. Interestingly, however, we did not observe, in preliminary experiments, a grossly altered DNA damage response or increased chromosomal instability in cell lines of our proband. There is a possible that the WRN carrier status in the patient reported here is incidental to his phenotype. It is also conceivable, however, that WRN heterozygosity and SAMHD1 homozygosity may have synergistically contributed to the atypical presentation and severe phenotype of this patient.

\section{ACKNOWLEDGMENTS}

We thank Ms. Lin Lee and Ms. Lindsey Theda for their technical assistances. This work was supported by grants from NIH (R24AG042328) from Ellison Medical Foundation (AG-SS-191507). Authors declare no conflict of interest.

\section{REFERENCES}

Beloglazova N, Flick R, Tchigvintsev A, Brown G, Popovic A, Nocek B, Yakunin AF. 2013. Nuclease activity of the human SAMHD1 protein implicated in the Aicardi-Goutieres syndrome and HIV-1 restriction. J Biol Chem 288:8101-8110.

Clifford R, Louis T, Robbe P, Ackroyd S, Burns A, Timbs AT, Colopy GW, Dreau H, Sigaux F, Judde JG, Rotger M, Telenti A, Lin YL, Pasero P, Maelfait J, Titsias M, Cohen DR, Henderson SJ, Ross M, Bentley D, Hillmen P, Pettitt A, Rehwinkel J, Knight SJ, Taylor JC, Crow YJ, Benkirane M, Schuh A. 2013. SAMHD1 is mutated recurrently in chronic lymphocytic leukemia and is involved in response to DNA damage. Blood

Dale RC, Gornall H, Singh-Grewal D, Alcausin M, Rice GI, Crow YJ. 2010. Familial Aicardi-Goutieres syndrome due to SAMHD1 mutations is associated with chronic arthropathy and contractures. Am J Med Genet A 152A:938-942.

Friedrich K, Lee L, Leistritz DF, Nurnberg G, Saha B, Hisama FM, Eyman DK, Lessel D, Nurnberg P, Li C, Garcia FVMJ, Kets CM, Schmidtke J, Cruz VT, Van den Akker PC, Boak J, Peter D, Compoginis G, Cefle K, Ozturk S, Lopez N, Wessel T, Poot M, Ippel PF, Groff-Kellermann B, Hoehn H, Martin GM, Kubisch C, Oshima J. 2010. WRN mutations in Werner syndrome patients: genomic rearrangements, unusual intronic mutations and ethnic-specific alterations. Hum Genet 128:103-111.

Lahouassa H, Daddacha W, Hofmann H, Ayinde D, Logue EC, Dragin L, Bloch N, Maudet C, Bertrand M, Gramberg T, Pancino G, Priet S, Canard B, Laguette N, Benkirane M, Transy C, Landau NR, Kim B, MargottinGoguet F. 2012. SAMHD1 restricts the replication of human immunodeficiency virus type 1 by depleting the intracellular pool of deoxynucleoside triphosphates. Nat Immunol 13:223-228.

Ogburn CE, Oshima J, Poot M, Chen R, Hunt KE, Gollahon KA, Rabinovitch PS, Martin GM. 1997. An apoptosis-inducing genotoxin differentiates heterozygotic carriers for Werner helicase mutations from wildtype and homozygous mutants. Human genetics 101:121-125.

Oshima J, Martin GM, Hisama FM. 2014. Werner Syndrome. In: Pagon RA, Adam MP, Bird TD, Dolan CR, Fong CT, Stephens K, editors GeneReviews. Seattle (WA).

Rice GI, Bond J, Asipu A, Brunette RL, Manfield IW, Carr IM, Fuller JC, Jackson RM, Lamb T, Briggs TA, Ali M, Gornall H, Couthard LR, Aeby A, Attard-Montalto SP, Bertini E, Bodemer C, Brockmann K, Brueton LA, Corry PC, Desguerre I, Fazzi E, Cazorla AG, Gener B, Hamel BC, Heiberg A, Hunter M, van der Knaap MS, Kumar R, Lagae L, Landrieu PG, Lourenco CM, Marom D, McDermott MF, van der Merwe W, Orcesi S, Prendiville JS, Rasmussen M, Shalev SA, Soler DM, Shinawi M, Spiegel R, Tan TY, Vanderver A, Wakeling EL, Wassmer E, Whittaker E, Lebon P, Stetson DB, Bonthron DT, Crow YJ. 2009. Mutations involved in Aicardi-Goutieres syndrome implicate SAMHD1 as regulator of the innate immune response. Nat Genet 41:829-832.

Stephenson JB. 2008. Aicardi-Goutieres syndrome (AGS). Eur J Paediatr Neurol 12:355-358.

Thiele H, du Moulin M, Barczyk K, George C, Schwindt W, Nurnberg G, Frosch M, Kurlemann G, Roth J, Nurnberg P, Rutsch F. 2010. Cerebral arterial stenoses and stroke: novel features of Aicardi-Goutieres syndrome caused by the Arg164X mutation in SAMHD1 are associated with altered cytokine expression. Hum Mutat 31:E1836-1850.

Xin B, Jones S, Puffenberger EG, Hinze C, Bright A, Tan H, Zhou A, Wu G, Vargus-Adams J, Agamanolis D, Wang H. 2011. Homozygous mutation in SAMHD1 gene causes cerebral vasculopathy and early onset stroke. Proc Natl Acad Sci U S A 108:5372-5377. 